\title{
Scintigraphy of Patient with Hematochezia
}

\author{
Brian Graner, Nichole Van Houten, and Helena Balon \\ Department of Radiology and Molecular Imaging, Oakland University and William Beaumont Health System, Royal Oak, Michigan
}

Gastrointestinal bleeding can result in significant morbidity. Scintigraphy plays an important role in detecting, localizing, and grading the bleed. Effective scintigraphic evaluation of gastrointestinal bleeding can be complicated by its intermittent nature and the patient's hemodynamic instability. Dynamic evaluation, delayed imaging, and an understanding of the labeling process are necessary tools to help improve detection rate and localization.

Key Words: gastrointestinal bleeding; in vitro RBC labeling; quality control

J Nucl Med Technol 2014; 42:79-80

DOI: $10.2967 /$ jnmt.113.132746

G astrointestinal bleeding can result in significant morbidity. Scintigraphy plays an important role in detecting, localizing, and grading the bleed. Dynamic evaluation, delayed imaging, and proper red blood cell (RBC) labeling are essential to have an effective impact on patient care.

\section{CASE REPORT}

An 83-y-old woman presented with hematochezia. The findings of a recent colonoscopy were negative, and the hemoglobin level was $7.7 \mathrm{~g} / \mathrm{dL}$.

A gastrointestinal bleed scan $(777 \mathrm{MBq}[21 \mathrm{mCi}]$ of RBCs labeled with ${ }^{99 \mathrm{~m}} \mathrm{Tc}$ in vitro) was acquired at $1 \mathrm{~min} /$ frame for $60 \mathrm{~min}$ (Fig. 1), with static images obtained at 70 and $80 \mathrm{~min}$ (Fig. 2).

No abnormal activity was identified during the initial dynamic acquisition (Fig. 1). The additional static imaging revealed radiotracer in the stomach and proximal small bowel (Fig. 2). A brisk prepyloric bleed was reported. Subsequent upper endoscopy revealed a gastric and esophageal ulcer.

\section{DISCUSSION}

The intermittent nature of gastrointestinal bleeding can complicate detection. Zettinig et al. reviewed 89

\footnotetext{
Received Sep. 17, 2013; revision accepted Oct. 23, 2013.

For correspondence or reprints contact: Brian Graner, Department of Radiology and Molecular Imaging, Oakland University and William Beaumont Health System, 3601 W. 13 Mile Rd., Royal Oak, MI 48073.

E-mail: brian.graner@beaumont.edu

Published online Jan. 27, 2014.

COPYRIGHT (C 2014 by the Society of Nuclear Medicine and Molecular Imaging, Inc.
}

gastrointestinal bleeding scans and reported that 30 of 41 positive scans $(73 \%)$ were positive on delayed imaging only (1). In another publication, Howarth also supported delayed imaging and added that dynamic imaging helps localize bleeding (2). Dynamic imaging improves the detection of small bleeds and helps differentiate a bleed from areas of hypervascularity or vascular anomalies.

When activity is identified within the stomach on a ${ }^{99 m} \mathrm{Tc}-$ $\mathrm{RBC}$ scan, poor labeling of the RBCs and free pertechnetate accumulating within the gastric mucosa must be considered. Imaging of the thyroid is essential. If the thyroid shows no uptake the bleed can be confirmed, unless the thyroid is surgically absent or was ablated, or the patient is on suppressive doses of thyroxine. Free pertechnetate can be secreted into the stomach lumen and will progress along the small bowel.

Another consideration with suspected gastric activity is radiotracer within superimposed structures, such as the transverse colon. Although each lumen has a distinct morphology, limited activity on initial imaging can make exact localization difficult. Delayed imaging helps in bleed localization by allowing recognition of structures into which antegrade or retrograde progression of activity occurs. In the presented case, imaging could not be continued because of hemodynamic instability.

Treatment of hemodynamic instability in the setting of anemia and suspected bleeding often includes transfusion. Labeling efficiency is affected by the presence of additives in the transfusion. The in-vitro UltraTag (Mallinckrodt Pharmaceuticals) kit labeling process involves adding anticoagulated blood to the kit containing stannous ion $\left(\mathrm{Sn}^{2+}\right)$. The stannous ion diffuses into the RBCs. After incubation, the extracellular $\mathrm{Sn}^{2+}$ must be oxidized to prevent it from reducing the extracellular ${ }^{99 \mathrm{~m}} \mathrm{Tc}-$ pertechnetate, because reduced ${ }^{99 \mathrm{~m} T c}$-pertechnetate is unable to enter the RBCs. ${ }^{99 \mathrm{~m}} \mathrm{Tc}$-pertechnetate is then added, and it diffuses into the pretinned RBCs. Once intracellular, pertechnetate is reduced by the intracellular $\mathrm{Sn}^{2+}$ and binds to hemoglobin.

In recently transfused patients, the ethylenediamine tetraacetic acid anticoagulant contained in the transfusion binds the stannous ion before it can enter the RBCs, significantly reducing the labeling efficiency $(3,4)$.

Finally, the amount of bleeding is also an important aspect of clinical decision making. Smith et al. investigated the use of "visual blood loss estimate" on delayed imaging (5). The bleed was graded as strongly versus weakly positive (tracer intensity greater than or less than the liver) and early versus late (time to positivity less than or greater than $1 \mathrm{~h}$ ). All 
FIGURE 1. Gastrointestinal bleed scan images acquired at $10 \mathrm{~min}(\mathrm{~A}), 30 \mathrm{~min}(\mathrm{~B})$, and $50 \mathrm{~min}(\mathrm{C})$.
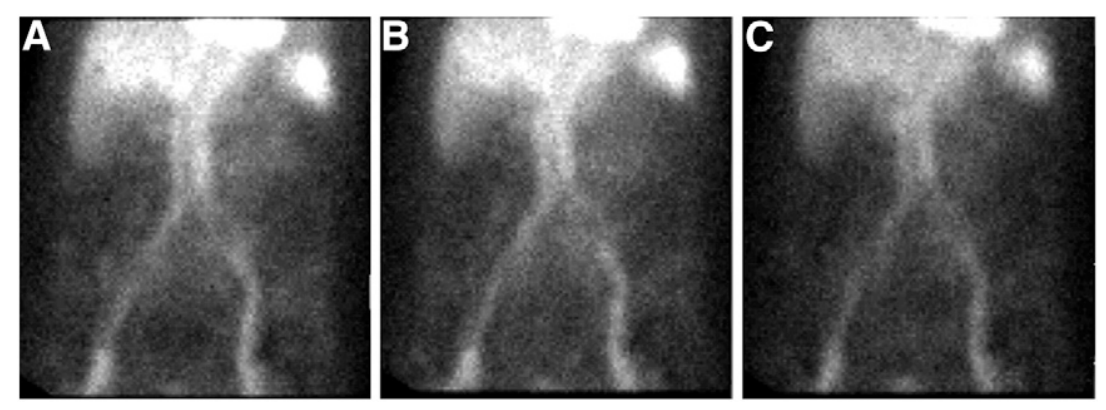

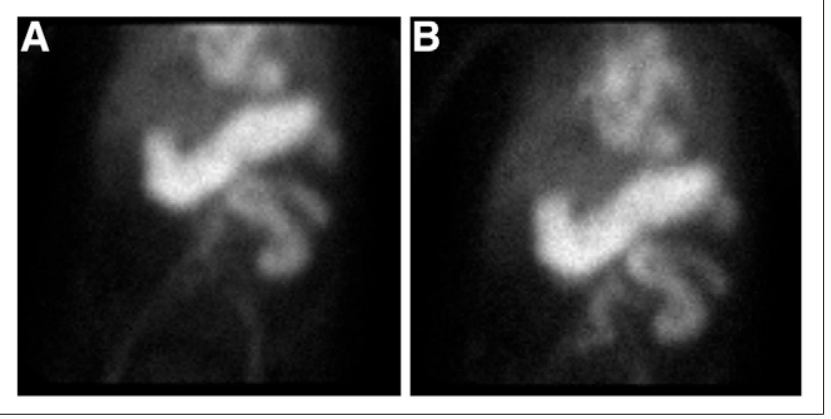

FIGURE 2. Static images acquired at $70 \mathrm{~min}(\mathrm{~A})$ and $80 \mathrm{~min}$ (B).

patients with early or strong positivity required aggressive management, as opposed to only $23 \%$ of those with late or weak positivity.

\section{CONCLUSION}

Effective scintigraphic evaluation of gastrointestinal bleeding can be complicated by its intermittent nature and the patient's hemodynamic instability. Dynamic evaluation, delayed imaging, and an understanding of the labeling process are necessary tools to help improve detection rate and localization.

\section{DISCLOSURE}

No potential conflict of interest relevant to this article was reported.

\section{REFERENCES}

1. Zettinig G, Staudenherz A, Leitha T. The importance of delayed images in gastrointestinal bleeding scintigraphy. Nucl Med Commun. 2002;23:803-808.

2. Howarth DM. The role of nuclear medicine in the detection of acute gastrointestinal bleeding. Semin Nucl Med. 2006;36:133-146.

3. Mock BH, Wellman HN. Stoichiometric Tc-99m labeling using stable kit solutions of stannous chloride and EDTA: concise communication. J Nucl Med. 1984;25:881-886.

4. Kuehne R, Reuter E. High RBC labeling efficiency by controlling pretinning with the modified in vivo/in vitro labeling method. J Nucl Med Technol. 1999;27:222226.

5. Smith R, Copely DJ, Bolen FH. ${ }^{99 m}$ Tc RBC scintigraphy: correlation of gastrointestinal bleeding rates with scintigraphic findings. AJR. 1987;148:869-874. 